\title{
REARRANGEMENTS AND TUNNELING SPLITTINGS IN SMALL WATER CLUSTERS
}

\author{
D. J. WALES \\ University Chemical Laboratories \\ Lensfield Road, Cambridge CB2 1EW, UK
}

\section{Introduction}

This paper was prepared in August 1997 for the proceedings volume of the NATO-ASI meeting on Recent Theoretical and Experimental Advances in Hydrogen Bonded Clusters edited by Sotiris Xantheas, which has so far failed to appear.

Recent far-infrared vibration-rotation tunneling (FIR-VRT) experiments [1-4] pose new challenges to theory because the interpretation and prediction of such spectra requires a detailed understanding of the potential energy surface (PES) away from minima. In particular we need a global description of the PES in terms of a complete reaction graph. Hence all the transition states and associated mechanisms which might give rise to observable tunneling splittings must be characterized. It may be possible to guess the detailed permutations of atoms from the transition state alone, but experience suggests this is unwise.

In this contribution a brief overview of the issues involved in treating the large amplitude motions of such systems will be given, with references to more detailed discussions and some specific examples. In particular we will consider the effective molecular symmetry group, the classification of rearrangement mechanisms, the location of minima and transition states and the calculation of reaction pathways. The application of these theories to small water clusters ranging from $\left(\mathrm{H}_{2} \mathrm{O}\right)_{2}$ to $\left(\mathrm{H}_{2} \mathrm{O}\right)_{6}$ will then be considered. More details can be found in recent reviews [5-8]. 


\section{The Effective Molecular Symmetry Group}

To classify the energy levels of systems executing large amplitude motions (non-rigid or 'floppy' molecules) we must look beyond point group symmetry. The PES contains all the permutational isomers of each stationary point. In the nomenclature of Bone et al. [9] we will refer to a structure as a particular molecular geometry and a version as a particular labelled permutational isomer of a given structure. Tunneling splittings occur when rovibronic wavefunctions localized in potential wells corresponding to different permutational isomers interfere with each other.

The Hamiltonian is invariant to arbitrary permutations of atoms of the same element, and to $E^{*}$, the inversion of all coordinates through the spacefixed origin. Molecular energy levels may therefore be classified according to irreducible representations (IR's) of the Complete Nuclear PermutationInversion (CNPI) group which is the direct product of the inversion group and the group containing all possible permutations of identical nuclei. The CNPI group is a true symmetry group of the full molecular Hamiltonian in the absence of external fields, and the elements are usually referred to as permutation-inversions (PI's). The CNPI group grows in size factorially with the number of equivalent nuclei, and rapidly becomes unwieldy. Fortunately, Longuet-Higgins [10] showed that it is sufficient to consider the subgroup of the CNPI group which contains only the PI's associated with barrierless processes and resolvable tunneling splittings. The associated rearrangement mechanisms are said to be feasible. For rigid molecules the MS group, of order $h_{\mathrm{RM}}$, is isomorphic to the point group [11,12], and all the associated PI's correspond to barrierless processes. Each rovibronic level is

$h_{\mathrm{CNPI}} / h_{\mathrm{RM}}$-fold degenerate because there are $h_{\mathrm{CNPI}} / h_{\mathrm{RM}}$ distinct versions on the PES [9] and each one supports an identical stack of energy levels.

A feasible rearrangement with a non-zero barrier leads to an enlargement of the MS group to include the new PI and all its products with the PI's of the rigid molecule MS group. Further feasible mechanisms are included in a similar fashion. The resulting MS group is a subgroup of the CNPI group obtained by removing all the PI's for a given reference version which are not feasible. The order of the MS group, $h_{\mathrm{MS}}$, and the order of the CNPI group, $h_{\mathrm{CNPI}}$, must satisfy $h_{\mathrm{CNPI}} / h_{\mathrm{MS}}=M$, where $M$ is an integer. The versions are then divided into $M$ disconnected sets. Each set contains $h_{\mathrm{MS}} / h_{\mathrm{RM}}$ distinct versions [9] all of which can be interconverted by one or more feasible operations.

To a first approximation the splitting pattern for any particular rovibronic level can be obtained from wavefunctions written as linear combinations of localized states which must transform according to IR's of the MS group. In this approach we apply degenerate perturbation theory to 
obtain a secular determinant whose eigenfunctions and eigenvalues are the required tunneling states and their energies. Mixing between different localized states is neglected in this approximation. Since the localized wavefunctions decay exponentially in the classically forbidden barrier regions it may be reasonable to make a Hückel-type approximation including non-zero offdiagonal matrix elements, $\beta$, only for minima which are directly connected by a feasible rearrangement. Even if the tunneling matrix elements are unknown it is still possible to deduce the likely splitting pattern along with symmetry classifications and nuclear spin statistics. This procedure has been automated using a computer program to calculate the reaction graph from a minimal set of generator PI's using double cosets [13] and similarity transforms [14].

\section{Classification of Rearrangement Mechanisms}

We define a degenerate rearrangement mechanism as one which links permutational isomers of the same structure via a single transition state [15]. Here we follow Murrell and Laidler's definition of a transition state as a stationary point with a single negative Hessian eigenvalue [16]. The largest tunneling splittings are expected for degenerate rearrangements (where the localized states are in resonance) with low barriers, short paths and small effective masses. Degenerate rearrangements can in turn be divided into two classes, namely symmetric degenerate rearrangements (SDR's) where the two sides of the path are related by a symmetry operation, and asymmetric degenerate rearrangements (ADR's) where they are not [17]. Most of the rearrangements discussed in the following sections are ADR's, which is likely to cause problems in choosing the fixed nodes for excited state calculations in the diffusion Monte Carlo (DMC) approach [18-21].

Recent experimental [22] and theoretical [23] studies of water trimer isotopomers both indicate that significant tunneling splittings occur in these species, even when the rearrangements involved are not strictly degenerate. (Within the Born-Oppenheimer approximation the isotopomers have the same PES, but the vibronic states are not in resonance between different wells.) We will also see in the following sections that tunneling splittings are observed for $\left(\mathrm{H}_{2} \mathrm{O}\right)_{4}$ and $\left(\mathrm{H}_{2} \mathrm{O}\right)_{6}$ where no suitable degenerate rearrangements have been found theoretically. In each case the tunneling may be the result of a series of steps mediated by true transition states, or perhaps even higher index saddles, where the end points are true permutational isomers. We suggest the term 'indirect tunneling' to describe this situation and distinguish it from 'non-degenerate tunneling' where the interaction is between states belonging to different structures. 


\section{Geometry Optimizations and Rearrangement Pathways}

Eigenvector-following provides a powerful technique for locating minima and transition states and calculating reaction pathways [24-29]. The precise algorithms employed in the present work have been described in detail elsewhere $[30,31]$. The step for eigendirection $i$ is

$$
h_{i}=\frac{ \pm 2 F_{i}}{\left|b_{i}\right|\left(1+\sqrt{1+4 F_{i}^{2} / b_{i}^{2}}\right)}, \quad\left\{\begin{array}{c}
+ \text { for walking uphill } \\
- \text { for walking downhill }
\end{array}\right\},
$$

where $b_{i}$ is the corresponding Hessian eigenvalue and $F_{i}$ is the component of the gradient in this direction. A separate trust radius is employed for each eigendirection by comparing the Hessian eigenvalue estimated from the current and previous gradients with the true value. Analytic first and second derivatives of the energy were used at every step.

In the $a b$ initio calculations these derivatives were mostly generated by the CADPAC program [32], and Cartesian coordinates were used throughout along with projection to remove overall rotation and translation [33,34]. Pathways were calculated by taking small displacements of $0.03 a_{0}$ away from a transition state both parallel and antiparallel to the transition vector, and then employing eigenvector-following energy minimization to find the associated minimum. The pathways obtained by this procedure have been compared to steepest-descent paths and pathways that incorporate a kinetic metric [35] in previous work - the mechanism is generally found to be correct [7]. Calculations employing rigid body intermolecular potentials were performed using the ORIENT3 program [36-38], which contains the same optimization package adapted for center-of-mass/orientational coordinates. Some of the calculations in the present work employ the ASP rigid water intermolecular potential of Millot and Stone [39] (somewhat modified from the published version) and the much simpler but widely-used TIP4P form $[40,41]$.

\section{Water Dimer}

The pioneering experiment of Dyke, Mack and Muenter [42] was followed by many experimental and theoretical studies [43-45] of $\left(\mathrm{H}_{2} \mathrm{O}\right)_{2}$ and $\left(\mathrm{D}_{2} \mathrm{O}\right)_{2}$. Dyke [46] classified the rovibronic energy levels in terms of permutationinversion group theory, and Coudert and Hougen used their internal-axis method and an empirical intermolecular potential to analyze the tunneling splittings theoretically [47-49]. A number of stationary points on the dimer PES were characterized by Smith et al. [50], including three true transition states. More recently the pathways corresponding to these three feasible rearrangements have been characterized [8]. Since the group theory of the 
Figure 1. 'Acceptor-tunneling' path calculated for $\left(\mathrm{H}_{2} \mathrm{O}\right)_{2}$. The first and last frames are the two minima, the middle frame is the transition state and three additional frames on each side of the path were selected to best illustrate the mechanism. A suitably scaled transition vector is superimposed on the transition state; this displacement vector lies parallel to the Hessian eigenvector corresponding to the unique negative eigenvalue.

water dimer is relatively well known, and has recently been summarized with reference to the true pathways [8], details will be omitted here. Two points of interest arise for the 'acceptor tunneling' pathway which leads to the largest splittings. First, this pathway was found to correspond to a 'methylamine-type' process [51] rather than a rotation about the local $C_{2}$ axis of the acceptor monomer (Figure 1) [8]. This result is in agreement with the analysis of Pugliano et al. for the ground state acceptor tunneling path based upon experiment [52]. 


\section{Water Trimer}

A flurry of experimental and theoretical studies [53-56] followed the initial FIR-VRT results of Pugliano and Saykally [57]. The energy level patterns observed in the most recent FIR-VRT experiments correspond to an oblate symmetric rotor with a large negative inertial defect, implying extensive out-of-plane motion of the non-hydrogen-bonded hydrogens. These results can be reconciled with the cyclic $C_{1}$ symmetry global minimum found in $a b$ initio calculations $[58,59]$ by vibrational averaging over large amplitude motions of the free (non-hydrogen-bonded) hydrogens on the timescale of the FIR-VRT experiment.

The vibrational averaging which leads to the oblate symmetric top spectrum is caused by the facile single flip mechanism. The corresponding transition state was probably first characterized by Owicki et al. [60] for an empirical potential. We have illustrated the mechanism before $[5,7,59]$ and readers are referred to these references for figures and a more detailed review of the literature. The single flip mechanism links each permutational isomer to two others in cyclic sets of six, giving a secular problem analogous to the $\pi$ system of benzene $[59,61]$ with splitting pattern:

$$
2 \beta_{\mathrm{f}}\left(A_{1}\right), \quad \beta_{\mathrm{f}}\left(E_{2}\right), \quad-\beta_{\mathrm{f}}\left(E_{1}\right), \quad-2 \beta_{\mathrm{f}}\left(A_{2}\right),
$$

where $\beta_{\mathrm{f}}$ is the tunneling matrix element for the flip. The MS group has order six and is isomorphic to $C_{3 h}$ [57].

The rearrangement responsible for the regular quartet splittings observed experimentally is probably the analogue of the 'donor tunneling' mechanism in the dimer, also known as 'bifurcation tunneling' because the transition state includes a bifurcated, double acceptor, double-donor arrangement [7]. When both the bifurcation mechanism and the single flip are feasible the MS group has order 48 [59]. However, there are six distinct ways for the bifurcation to occur with accompanying flips of neighbouring monomers, and these give two distinct splitting patterns [7]. The most recent experiments show that some of the quartets are further split by Coriolis coupling $[62,63]$. Entirely regular quartets are found to be associated with generators containing $E^{*}$ in the latter studies [7,63].

A more detailed account of the above mechanisms, group theory and quantum dynamics calculations can be found elsewhere $[5,7]$. The analysis of different bifurcation and flip mechanisms is considered in more detail below for the water pentamer.

\section{Water Tetramer}

A symmetric doublet splitting of $5.6 \mathrm{MHz}$ has been reported in two FIRVRT experiments for $\left(\mathrm{D}_{2} \mathrm{O}\right)_{4}[64,65]$. The cyclic global minimum of the 
tetramer has $S_{4}$ symmetry [66-69] and lacks the 'frustration' exhibited by the cyclic trimer and pentamer. Schütz and coworkers found no true transition states correpsonding to degenerate rearrangements in the torsional space of the tetramer [70,71]. A more systematic survey revealed many more non-degenerate rearrangement mechanisms and just one true degenerate rearrangement of the global minimum, which disappeared in correlated calculations [72].

The effective MS group is isomorphic to $C_{2 v}$ for an overall quadruple flip [64] mediated by any pathway which gives the same effective generator PI [73]. There are at least three possible routes involving (1) a concerted quadruple flip (via an index four saddle), (2) true transition states and local minima, or (3) index two saddles and local minima [70,72]. The splitting pattern for the only direct degenerate rearrangement found so far for this cluster is more complex [72]. Four-dimensional quantum calculations of torsional vibrational states with a model Hamiltonian suggest that a stepwise pathway via the true transition states may contribute most to tunneling [74], but there are too many approximations involved for a definitive conclusion to be drawn. The possibility appears to remain that the observed splittings are due to 'indirect tunneling', as defined in §3. A more detailed discussion and illustrations of the mechanisms have been given elsewhere [72].

TABLE 1. Generators and splitting patterns for the 15 distinct bifurcation+flip combinations considered for water pentamer in $\S 8$. See also Figure 2.

\begin{tabular}{lllc}
\hline Description & generator & inverse & pattern \\
\hline A-bif & $($ ABCDE $)(13579246810)^{*}$ & E-bif & A \\
A-bif+BCD-flips & $($ AEDCB $)(19753210864)$ & B-bif+CDE-flips & A \\
E-bif+BCD-flips & $($ ABCDE $)(13571024689)$ & D-bif+ABC-flips & A \\
D-bif+BCE-flips & $($ ABCDE $)(13581024679)$ & C-bif+ABD-flips & A \\
B-bif+ACD-flips & $($ AEDBC $)(19754210863)$ & C-bif+BCD-flips & A \\
A-bif+B-flip & $($ ACEBD $)(15937261048)$ & D-bif+E-flip & B \\
A-bif+BC-flips & $($ ADBEC $)(17395284106)^{*}$ & C-bif+DE-flips & B \\
E-bif+D-flip & $($ ADBEC $)(17310628495)$ & B-bif+A-flip & B \\
E-bif+CD-flips & $($ ACEBD $)(17310628495)^{*}$ & C-bif+AB-flips & B \\
D-bif+EC-flips & $($ ACEBD $)(15938261047)^{*}$ & B-bif+AC-flips & B \\
A-bif+BCDE-flips & $(12)^{*}$ & self-inverse & C \\
E-bif+ABCD-flips & $(910)^{*}$ & self-inverse & C \\
D-bif+ABCE-flips & $(78)^{*}$ & self-inverse & C \\
B-bif+ACDE-flips & $(34)^{*}$ & self-inverse & C \\
C-bif+ABDE-flips & $(56)^{*}$ & self-inverse & C \\
\hline
\end{tabular}


Figure 2. The effect of A-bifurcation and A-bifurcation+B-flip on a reference version of the water pentamer. The arrows indicate the direction of hydrogen-bond donation. The generator PI is deduced by putting the structure in coincidence with the reference, with an intervening inversion if necessary.

\section{Water Pentamer}

The water pentamer exhibits a number of similarities to the trimer due to the frustrated cyclic global minimum. The first FIR-VRT results for $\left(\mathrm{D}_{2} \mathrm{O}\right)_{5}$ did not reveal any tunneling splittings [75]. However, analogues of the single flip and bifurcation mechanisms, described above for the trimer, have been found along with a number of pathways connecting higher energy minima $[31,76]$. Both the flip and the bifurcation mechanism considered in isolation produce an MS group of order 10 isomorphic to $C_{5 h}$ with each version connected to two others in a cyclic reaction graph containing 10 versions. The predicted splitting pattern in the simplest Hückel approximation is the same as for the $\pi$-system of 10-annulene (cyclodecapentaene):

$$
2 \beta\left(A^{\prime}\right), \quad \phi \beta\left(E_{2}^{\prime \prime}\right), \quad \phi^{-1} \beta\left(E_{1}^{\prime}\right), \quad-\phi^{-1} \beta\left(E_{1}^{\prime \prime}\right), \quad-\phi \beta\left(E_{2}^{\prime}\right), \quad-2 \beta\left(A^{\prime \prime}\right),
$$

where $\beta$ is the appropriate tunneling matrix element, $\phi=(\sqrt{5}+1) / 2$ is the golden ratio and $\phi^{-1}=(\sqrt{5}-1) / 2=1 / \phi$. The symmetry species in parentheses are appropriate if the generator corresponds to the operation $S_{5}$ of $C_{5 h}$. If both the flip and bifurcation mechanisms are feasible then the MS group increases in dimension to order 320 and the splitting pattern becomes rather complicated [31]. Qualitative estimates for the magnitude 
of the splittings suggested that the flip should lead to observable effects in both $\left(\mathrm{H}_{2} \mathrm{O}\right)_{5}$ and $\left(\mathrm{D}_{2} \mathrm{O}\right)_{5}$, while bifurcation tunneling might produce splittings at the limit of current resolution [31]. The latest experimental results appear to be in agreement with these predictions [77].

TABLE 2. Splitting pattern type $\mathrm{C}$ for five of the possible pentamer bifurcation/flip combinations. $\phi=(\sqrt{5}+1) / 2$.

\begin{tabular}{|c|c|c|c|c|c|}
\hline level & energy & symmetry & level & energy & symmetry \\
\hline 1 & $2 \beta_{f}+\beta_{b}$ & $A_{1}^{+}$ & 56 & $-2 \beta_{f}-\beta_{b}$ & $A_{1}^{-}$ \\
\hline 2 & $2 \beta_{f}+0.603 \beta_{b}$ & $H_{6}^{+}$ & 55 & $-2 \beta_{f}-0.603 \beta_{b}$ & $H_{6}^{-}$ \\
\hline 3 & $2 \beta_{f}+0.206 \beta_{b}$ & $H_{1}^{+}$ & 54 & $-2 \beta_{f}-0.206 \beta_{b}$ & $H_{1}^{-}$ \\
\hline 4 & $2 \beta_{f}+0.203 \beta_{b}$ & $H_{2}^{+}$ & 53 & $-2 \beta_{f}-0.203 \beta_{b}$ & $H_{2}^{-}$ \\
\hline 5 & $2 \beta_{f}-0.194 \beta_{b}$ & $H_{3}^{+}$ & 52 & $-2 \beta_{f}+0.194 \beta_{b}$ & $H_{3}^{-}$ \\
\hline 6 & $2 \beta_{f}-0.197 \beta_{b}$ & $H_{4}^{+}$ & 51 & $-2 \beta_{f}+0.197 \beta_{b}$ & $H_{4}^{-}$ \\
\hline 7 & $2 \beta_{f}-0.597 \beta_{b}$ & $H_{5}^{+}$ & 50 & $-2 \beta_{f}+0.597 \beta_{b}$ & $H_{5}^{-}$ \\
\hline 8 & $2 \beta_{f}-\beta_{b}$ & $A_{2}^{+}$ & 49 & $-2 \beta_{f}+\beta_{b}$ & $A_{2}^{-}$ \\
\hline 9 & $\phi \beta_{f}+\beta_{b}$ & $E_{4}^{-} \oplus H_{5}^{-}$ & 48 & $-\phi \beta_{f}-\beta_{b}$ & $E_{4}^{+} \oplus H_{5}^{+}$ \\
\hline 10 & $\phi \beta_{f}+0.848 \beta_{b}$ & $H_{3}^{-}$ & 47 & $-\phi \beta_{f}-0.848 \beta_{b}$ & $H_{3}^{+}$ \\
\hline 11 & $\phi \beta_{f}+0.451 \beta_{b}$ & $H_{1}^{-} \oplus H_{4}^{-}$ & 46 & $-\phi \beta_{f}-0.451 \beta_{b}$ & $H_{1}^{+} \oplus H_{4}^{+}$ \\
\hline 12 & $\phi \beta_{f}+0.204 \beta_{b}$ & $H_{5}^{-}$ & 45 & $-\phi \beta_{f}-0.204 \beta_{b}$ & $H_{5}^{+}$ \\
\hline 13 & $\phi \beta_{f}+0.050 \beta_{b}$ & $H_{2}^{-}$ & 44 & $-\phi \beta_{f}-0.050 \beta_{b}$ & $H_{2}^{+}$ \\
\hline 14 & $\phi \beta_{f}-0.044 \beta_{b}$ & $H_{4}^{-}$ & 43 & $-\phi \beta_{f}+0.044 \beta_{b}$ & $H_{4}^{+}$ \\
\hline 15 & $\phi \beta_{f}-0.196 \beta_{b}$ & $H_{6}^{-}$ & 42 & $-\phi \beta_{f}+0.196 \beta_{b}$ & $H_{6}^{+}$ \\
\hline 16 & $\phi \beta_{f}-0.444 \beta_{b}$ & $H_{2}^{-} \oplus H_{3}^{-}$ & 41 & $-\phi \beta_{f}+0.444 \beta_{b}$ & $H_{2}^{+} \oplus H_{3}^{+}$ \\
\hline 17 & $\phi \beta_{f}-0.846 \beta_{b}$ & $H_{1}^{-}$ & 40 & $-\phi \beta_{f}+0.846 \beta_{b}$ & $H_{1}^{+}$ \\
\hline 18 & $\phi \beta_{f}-\beta_{b}$ & $E_{2}^{-} \oplus H_{6}^{-}$ & 39 & $-\phi \beta_{f}+\beta_{b}$ & $E_{2}^{+} \oplus H_{6}^{+}$ \\
\hline 19 & $\phi^{-1} \beta_{f}+\beta_{b}$ & $E_{1}^{+} \oplus H_{6}^{+}$ & 38 & $-\phi^{-1} \beta_{f}-\beta_{b}$ & $E_{1}^{-} \oplus H_{6}^{-}$ \\
\hline 20 & $\phi^{-1} \beta_{f}+0.847 \beta_{b}$ & $\mathrm{H}_{2}^{+}$ & 37 & $-\phi^{-1} \beta_{f}-0.847 \beta_{b}$ & $\mathrm{H}_{2}^{-}$ \\
\hline 21 & $\phi^{-1} \beta_{f}+0.451 \beta_{b}$ & $H_{1}^{+} \oplus H_{4}^{+}$ & 36 & $-\phi^{-1} \beta_{f}-0.451 \beta_{b}$ & $H_{1}^{-} \oplus H_{4}^{-}$ \\
\hline 22 & $\phi^{-1} \beta_{f}+0.201 \beta_{b}$ & $H_{6}^{+}$ & 35 & $-\phi^{-1} \beta_{f}-0.201 \beta_{b}$ & $H_{6}^{-}$ \\
\hline 23 & $\phi^{-1} \beta_{f}-0.042 \beta_{b}$ & $H_{3}^{+}$ & 34 & $-\phi^{-1} \beta_{f}+0.042 \beta_{b}$ & $H_{3}^{-}$ \\
\hline 24 & $\phi^{-1} \beta_{f}-0.053 \beta_{b}$ & $\overrightarrow{H_{1}^{+}}$ & 33 & $-\phi^{-1} \beta_{f}+0.053 \beta_{b}$ & $H_{1}^{-}$ \\
\hline 25 & $\phi^{-1} \beta_{f}-0.199 \beta_{b}$ & $H_{4}^{+}$ & 32 & $-\phi^{-1} \beta_{f}+0.199 \beta_{b}$ & $H_{5}^{-}$ \\
\hline 26 & $\phi^{-1} \beta_{f}-0.444 \beta_{b}$ & $H_{2}^{+} \oplus H_{3}^{+}$ & 31 & $-\phi^{-1} \beta_{f}+0.444 \beta_{b}$ & $H_{2}^{-} \oplus H_{3}^{-}$ \\
\hline 27 & $\phi^{-1} \beta_{f}-0.847 \beta_{b}$ & $H_{4}^{+}$ & 30 & $-\phi^{-1} \beta_{f}+0.847 \beta_{b}$ & $H_{4}^{-}$ \\
\hline 28 & $\phi^{-1} \beta_{f}-\beta_{b}$ & $E_{3}^{+} \oplus H_{5}^{+}$ & 29 & $-\phi^{-1} \beta_{f}+\beta_{b}$ & $E_{3}^{-} \oplus H_{5}^{-}$ \\
\hline
\end{tabular}

Since new experiments suggest that bifurcation tunneling splittings might be resolvable for $\left(\mathrm{H}_{2} \mathrm{O}\right)_{5}$ we will now consider the group theory in more detail to illustrate how such analyses are performed. The labelling scheme and assignment of generator PI's is illustrated for two possible bi- 
furcation+flip combinations in Figure 2. The labelling scheme in the figure enables us to identify all the possible bifurcation/flip processes which correspond to degenerate rearrangements using the letters $\mathrm{A}-\mathrm{E}$ to label the five monomers. Here we exclude processes where the bifurcating monomer also flips. The bifurcation can occur at any of the five monomers, and in each case the frustrated pair of neighbouring free hydrogens can appear on any of the five edges. This gives a total of 25 bifurcation/flip combinations. Five of the resulting generators are self-inverse while the other twenty fall into non-self-inverse pairs to give a total of 15 distinct mechanisms (Table 1). For the same labelling scheme the generator for the single flip is $(\mathrm{ABCDE})(13579)(246810)^{*}$ with inverse (AEDCB)(19753)(210864)*.

Previously we identified two splitting patterns for different bifurcation/flip combinations in the pentamer, and labelled these A and B. In the present systematic analysis a third splitting pattern, type $\mathrm{C}$, emerges, as shown in Table 2. All 15 combinations result in the same MS group, $G(320)$, described previously [31]. The C-type pattern occurs for the five generators of the form $(i j)^{*}$; for the trimer such generators also correspond to one particular splitting pattern [7]. The A-type pattern is found for the bifurcation accompanied by zero or three flips and the B-type pattern is found for the bifurcation accompanied by one or two flips (Table 1). All three patterns include four sets of ten lines and two sets of eight; they all involve irregular submultiplets. In the $\mathrm{C}$ pattern every energy level $\lambda$ has a partner with energy $-\lambda$ (and opposite parity with respect to $E^{*}$ ), implying that there are no odd-membered rings in the reaction graph [78]. The splittings within each manifold with a constant coefficient of $\beta_{f}$ (the tunneling matrix element for the flip) also obey an approximate pairing rule.

\section{Water Hexamer}

On the basis of an isotope mixture test Liu et al. [79] have assigned a VRT band of $\left(\mathrm{H}_{2} \mathrm{O}\right)_{6}$ at $83 \mathrm{~cm}^{-1}$. For this cluster a number of different structures lie rather close in energy, with the four lowest energy isomers reported by Tsai and Jordan separated by only about $100 \mathrm{~cm}^{-1}$ [80]. The most accurate $a b$ initio calculations performed to date suggest that a 'cage' structure lies lowest, followed closely by 'prism' and 'book' forms [81]. The assignment

of the experimental spectrum to the cage isomer was made on the basis of DMC calculations of the vibrationally averaged rotational constants [79]. It has been suggested [79] that a single structure is observed because the clusters have very low internal temperatures (around $5 \mathrm{~K}$ ) under experimental conditions. However, each experimental line is split into a triplet with intensity ratio 9:6:1 and separation $1.92 \mathrm{MHz}$. Liu et al. have explained how this pattern might arise from two hypothetical degenerate rearrangements 
Figure 3. Single flip mechanism which interconverts hexamer cage isomers C1 and C2.

of monomers in similar chemical environments [79]. However, no low-lying degenerate rearrangements have been found in studies employing both empirical and $a b$ initio calculations $[8,82,83]$. Four isomers of the cage have been found independently with very similar ASP-type potentials $[8,82,83]$ and checked in $a b$ initio calculations [82,83]. Flip and bifurcation rearrangements of the terminal single-donor, single-acceptor monomers exist which interconvert 16 versions of the cage structure, including four versions of each low-lying isomer [8]. Two examples are shown in Figures 3 and 4.

The resulting MS group can be found by considering the effective generators for combinations of flip and bifurcation processes which together result in a permutation of the same structure [73]. The same group is obtained for all four of the cage isomers; it contains four elements and is isomorphic to $C_{2 v}$ [8]. The effective reaction graph for one of the four cage isomers is shown in Figure 5. The four versions are connected in a cyclic 
Figure 4. Bifurcation mechanism which interconverts hexamer cage isomers C1 and C2.

reaction graph so that the simplest Hückel $\pi$ treatment gives a splitting pattern equivalent to that of the $\pi$ system in cyclobutadiene:

$$
2 \beta\left(A_{1}\right), \quad 0\left(A_{2}, B_{1}\right), \quad-2 \beta\left(B_{2}\right),
$$

where the symmetry labels are appropriate for a particular correspondence between the PI's and the elements of $C_{2 v}$ [8]. The accidental degeneracy of the $A_{2}$ and $B_{1}$ states would be broken at higher resolution because the $\beta$ matrix elements connecting the four versions of each isomer are all slightly different. The relative nuclear spin weights for rovibronic states are 9:3:3:1 for $\left(\mathrm{H}_{2} \mathrm{O}\right)_{6}$ and $4: 2: 2: 1$ for $\left(\mathrm{D}_{2} \mathrm{O}\right)_{6}$ corresponding to $A_{1}: A_{2}: B_{1}: B_{2}$. If the accidental degeneracy is unresolved then the relative intensities of the three triplet components would be 9:6:1 for $\left(\mathrm{H}_{2} \mathrm{O}\right)_{6}$ and 4:4:1 for $\left(\mathrm{D}_{2} \mathrm{O}\right)_{6}$. This result is equivalent to that obtained by Liu et al. [79] but does not require the existence of any hypothetical mechanisms. A similar analysis 
Figure 5. The effective reaction graph for four permutational isomers of cage structure C1 which are linked by stepwise flip and bifurcation rearrangements involving different cage structures.

holds for all four cage isomers. However, if this explanation is correct then it seems likely that all four cage isomers would be present under experimental conditions, which seems to be incompatible with the interpretation of the spectrum in terms of a single isomer [79]. Unless low energy degenerate rearrangements of the cage isomers are found the above 'indirect tunneling' mechanism seems to be the most plausible explanation that theory can provide.

Two-dimensional quantum calculations of torsional vibrational states for the single-acceptor, single-donor monomers have also been carried out [8]. The other four monomers are held fixed in this model. Both TIP4P $[40,41]$ and ASP potentials [39] were considered. For TIP4P all the wavefunctions were delocalized over the torsional degrees of freedom of the two monomers. However, for the ASP potential the ground state was found to be localized, in agreement with DMC calculations of Gregory and Clary [83]. The latter result appears to be incompatible with the experimental observation of tunneling splittings. However, the two-dimensional calculation suggests that there exist low-lying vibrational states which are delocalized over more than one cage isomer. More details, along with illustrations of the wavefunctions, can be found elsewhere [8]. These results provide a clear illustration of how tunneling splittings can be sensitive to the excitation of particular vibrational modes. Since experiment measures the difference between ground and excited state splittings a direct comparison with theory is often difficult.

\section{References}

1. R. C. Cohen and R. J. Saykally, J. Phys. Chem. 94:7991 (1990)

2. N. Pugliano and R. J. Saykally, J. Chem. Phys. 96:1832 (1992)

3. R. J. Saykally and G. A. Blake, Science 259:1570 (1993) 
4. K. Liu, J. D. Cruzan and R. J. Saykally, Science 271:929 (1996)

5. D. J. Wales in: Advances in Molecular Vibrations and Collision Dynamics, Z. Bačić and J. Bowman, eds., JAI Press, Greenwich (1997)

6. D. J. Wales in: Encyclopedia of Computational Chemistry, P. v. R. Schleyer, ed., Wiley, New York (1997)

7. T. R. Walsh and D. J. Wales, J. Chem. Soc., Faraday Trans. 92:2505 (1996)

8. D. J. Wales in: Theory of Atomic and Molecular Clusters, J. Jellinek, ed., SpringerVerlag, Berlin (1998)

9. R. G. A. Bone, T. W. Rowlands, N. C. Handy and A. J. Stone, Molec. Phys. 72:33 (1991)

10. H. C. Longuet-Higgins, Molec. Phys. 6:445 (1963)

11. J. T. Hougen, J. Chem. Phys. 37:1433 (1962)

12. J. T. Hougen, J. Chem. Phys. 39:358 (1962)

13. W. Hasselbarth and E. Ruch, Theo. Chim. Acta 29:259 (1973)

14. D. J. Wales, J. Amer. Chem. Soc. 115:11191 (1993)

15. R. E. Leone and P. v. R. Schleyer, Angew. Chem. Int. Ed. Engl., 9:860 (1970)

16. J. N. Murrell and K. J. Laidler, J. Chem. Soc., Faraday II 64:371 (1968)

17. J. G. Nourse, J. Amer. Chem. Soc. 102:4883 (1980)

18. J. B. Anderson, J. Chem. Phys. 63:1499 (1975)

19. J. K. Gregory and D. C. Clary, J. Chem. Phys. 103:8924 (1995)

20. J. K. Gregory and D. C. Clary, J. Chem. Phys. 102:7817 (1995)

21. J. K. Gregory and D. C. Clary, J. Chem. Phys. 105:6626 (1996)

22. K. Liu, M. G. Brown, M. R. Viant, J. D. Cruzan and R. J. Saykally, Molec. Phys. 89:1373 (1996)

23. D. Sabo, Z. Bačić, G. Stephan, S. Leutwyler, Chem. Phys. Lett. 261:318 (1996)

24. C. J. Cerjan and W. H. Miller, J. Chem. Phys. 75:2800 (1981)

25. J. Simons, P. Jørgenson, H. Taylor and J. Ozment, J. Phys. Chem. 87:2745 (1983)

26. D. O'Neal, H. Taylor and J. Simons, J. Phys. Chem. 88:1510 (1984)

27. A. Banerjee, N. Adams, J. Simons and R. Shepard, J. Phys. Chem. 89:52 (1985)

28. J. Baker, J. Comput. Chem. 7:385 (1986)

29. J. Baker, J. Comput. Chem. 8:563 (1987)

30. D. J. Wales, J. Chem. Phys. 101:3750 (1994)

31. D. J. Wales and T. R. Walsh, J. Chem. Phys. 105:6957 (1996)

32. R. D. Amos and J. E. Rice, CADPAC: the Cambridge Analytic Derivatives Package, Issue 4.0; Cambridge (1987)

33. J. Baker and W. H. Hehre, J. Comp. Chem., 12:606 (1991)

34. M. Page and J. W. McIver, J. Chem. Phys. 88:922 (1988)

35. A. Banerjee and N. P. Adams, Int. J. Quant. Chem. 43:855 (1992)

36. P. L. A. Popelier, A. J. Stone and D. J. Wales, J. Chem. Soc., Faraday Discuss. 97:243 (1994)

37. D. J. Wales, P. L. A. Popelier and A. J. Stone, J. Chem. Phys. 102:5556 (1995)

38. D. J. Wales, A. J. Stone and P. L. A. Popelier, Chem. Phys. Lett. 240:89 (1995)

39. C. Millot and A. J. Stone, Molec. Phys. 77:439 (1992)

40. W. L. Jorgensen, J. Amer. Chem. Soc. 103:335 (1981)

41. W. L. Jorgensen, J. Chandraesekhar, J. W. Madura, R. W. Impey and M. L. Klein, J. Chem. Phys. 79:926 (1983)

42. T. R. Dyke, K. M. Mack and J. S. Muenter, J. Chem. Phys. 66:498 (1977)

43. G. T. Fraser, Int. Rev. Phys. Chem. 10:189 (1991)

44. S. Scheiner, Annu. Rev. Phys. Chem. 45:23 (1994)

45. N. Pugliano, J. D. Cruzan, J. G. Loeser and R. J. Saykally, J. Chem. Phys. 98:6600 (1993)

46. T. R. Dyke, J. Chem. Phys. 66:492 (1977)

47. J. T. Hougen, J. Mol. Spectr. 114:395 (1985)

48. L. H. Coudert, J. T. Hougen, J. Mol. Spectr. 130:86 (19880

49. L. H. Coudert, J. T. Hougen, J. Mol. Spectr. 139:259 (1990) 
50. B. J. Smith, D. J. Swanton, J. A. Pople, H. F. Schaefer and L. Radom, J. Chem. Phys. 92:1240 (1990)

51. M. Tsuboi, A. Y. Hirakawa, T. Ino, Y. Sasaki, K. Tamagake, J. Chem. Phys. 41:2721 (1964)

52. N. Pugliano, J. D. Cruzan, J. G. Loeser and R. J. Saykally, J. Chem. Phys. 98:6600 (1993)

53. S. Suzuki and G. A. Blake, Chem. Phys. Lett. 229:499 (1994)

54. K. Liu, J. G. Loeser, M. J. Elrod, B. C. Host, J. A. Rzepiela, N. Pugliano and R. J. Saykally, J. Amer. Chem. Soc. 116:3507 (1994)

55. K. Liu, M. J. Elrod, J. G. Loeser, J. D. Cruzan, M. Brown and R. J. Saykally, Faraday Discuss. Chem. Soc. 97:35 (1994)

56. D. Sabo, Z. Bačić, T. Bürgi and S. Leutwyler, Chem. Phys. Lett. 244:283 (1995)

57. N. Pugliano and R. J. Saykally, Science 257:1937 (1992)

58. J. Del Bene and J. A. Pople, J. Chem. Phys. 58:3605 (19730

59. D. J. Wales, J. Amer. Chem. Soc. 115:11180 (1993)

60. J. C. Owicki, L. L. Shipman and H. A. Scheraga, J. Phys. Chem. 79:1794 (1975)

61. T. Bürgi, S. Graf, S. Leutwyler and W. Klopper, J. Chem. Phys. 103:1077 (1995)

62. A. van der Avoird, E. H. T. Olthof and P. E. S. Wormer, J. Chem. Phys. 105:8034 (1996)

63. E. H. T. Olthof, A. van der Avoird, P. E. S. Wormer, K. Liu and R. J. Saykally, J. Chem. Phys. 105:8051 (1996)

64. J. D. Cruzan, L. B. Braly, K. Liu, M. G. Brown, J. G. Loeser and R. J. Saykally,Science 271:59 (1996)

65. J. D. Cruzan, M. G. Brown, K. Liu, L. B. Braly, R. J. Saykally, J. Chem. Phys. 105:6634 (1996)

66. J. Del Bene and J. A. Pople, J. Chem. Phys. 52:4858 (19700

67. B. R. Lentz and H. A. Scheraga, J. Chem. Phys. 58:5296 (1973)

68. H. Kistenmacher, G. C. Lie, H. Popkie and E. Clementi, J. Chem. Phys. 61:546 (1974)

69. K. S. Kim, M. Dupuis, G. C. Lie and E. Clementi, Chem. Phys. Lett. 131:451 (1986)

70. M. Schütz, W. Klopper, H. P. Luthi and S. Leutwyler, J. Chem. Phys. 103:6114 (1995)

71. O. Engkvist, N. Forsberg, M. Schütz and G. Karlström, Mol. Phys. 90:277 (1997)

72. D. J. Wales and T. R. Walsh, J. Chem. Phys. 106:7193 (1997)

73. B. J. Dalton and P. D. Nicholson, Int. J. Quantum Chem. 9:325 (1975)

74. T. R. Walsh and D. J. Wales, in preparation

75. K. Liu, M. G. Brown, J. D. Cruzan and R. J. Saykally, Science 271:62 (1996)

76. D. J. Wales, Science 271:925 (1996)

77. K. Liu, M. G. Brown, J. D. Cruzan and R. J. Saykally, J. Phys. Chem. A 101:9011 (1997)

78. C. A. Coulson and S. Rushbrooke, Proc. Camb. Phil. Soc. 36:193 (1940)

79. K. Liu, M. G. Brown, C. Carter, R. J. Saykally, J. K. Gregory and D. C. Clary, Nature 381:501 (1996)

80. C. J. Tsai and K. D. Jordan, Chem. Phys. Lett. 213:181 (1993)

81. K. Kim, K. Jordan and T. S. Zwier, J. Amer. Chem. Soc. 116:11568 (1994)

82. J. K. Gregory and D. C. Clary in: Advances in Molecular Vibrations and Collision Dynamics, Z. Bačić and J. Bowman, eds., JAI Press, Greenwich (1997)

83. J. K. Gregory and D. C. Clary, J. Phys. Chem. A 101:6813 (1997) 

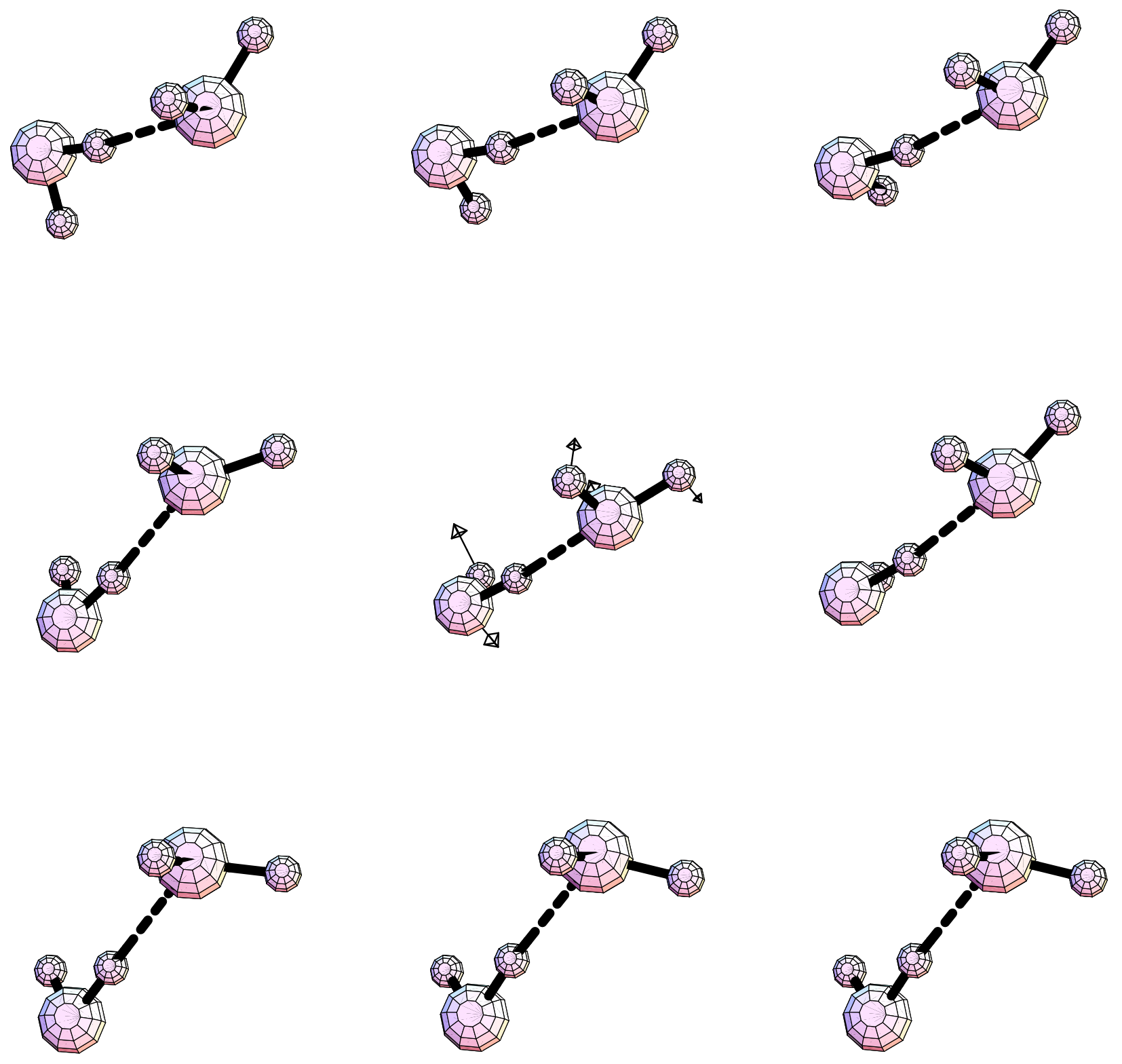

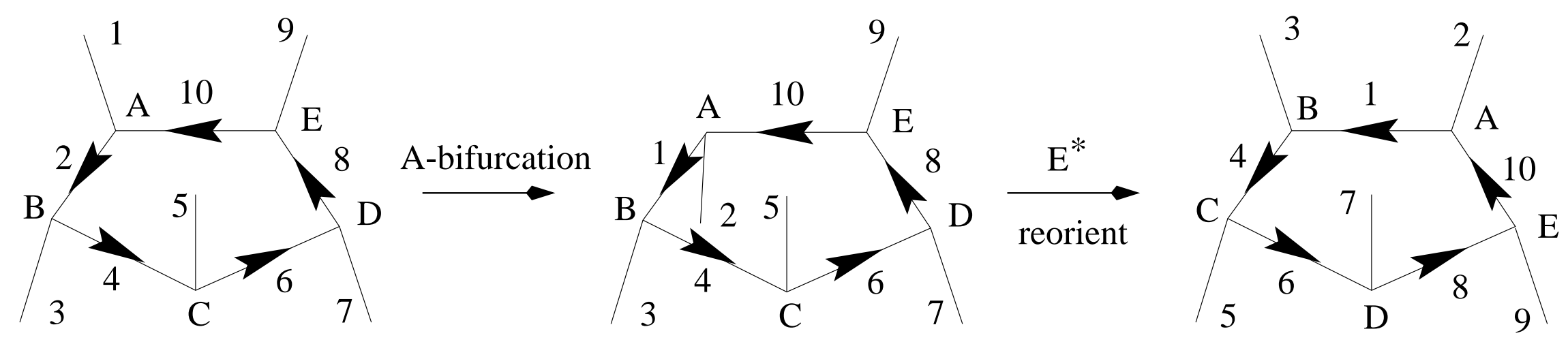

generator $=(\mathrm{ABCDE})(13579246810)^{*}$
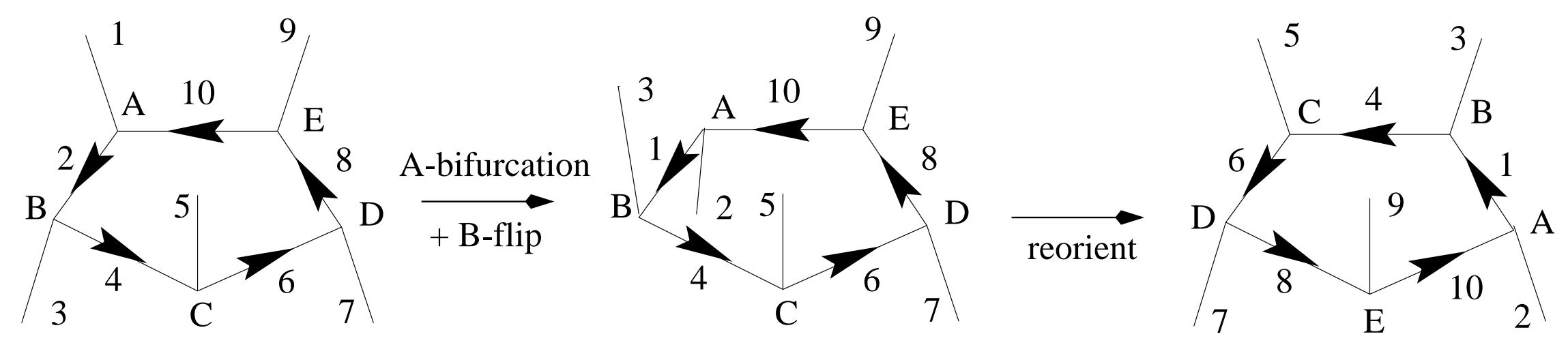

generator $=(A C E B D)(15937261048)$ 

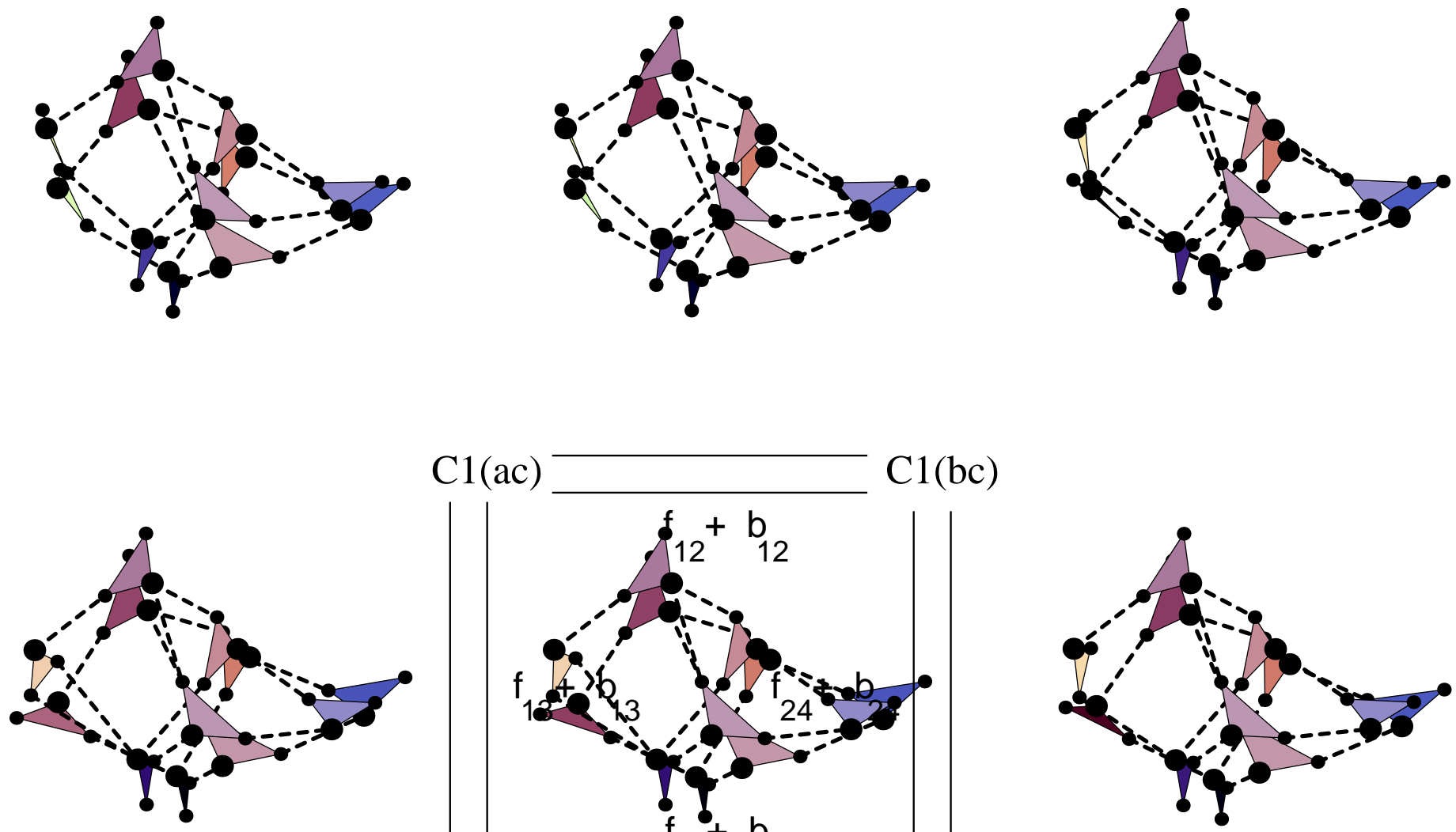

C1(ac)

C1(bc)
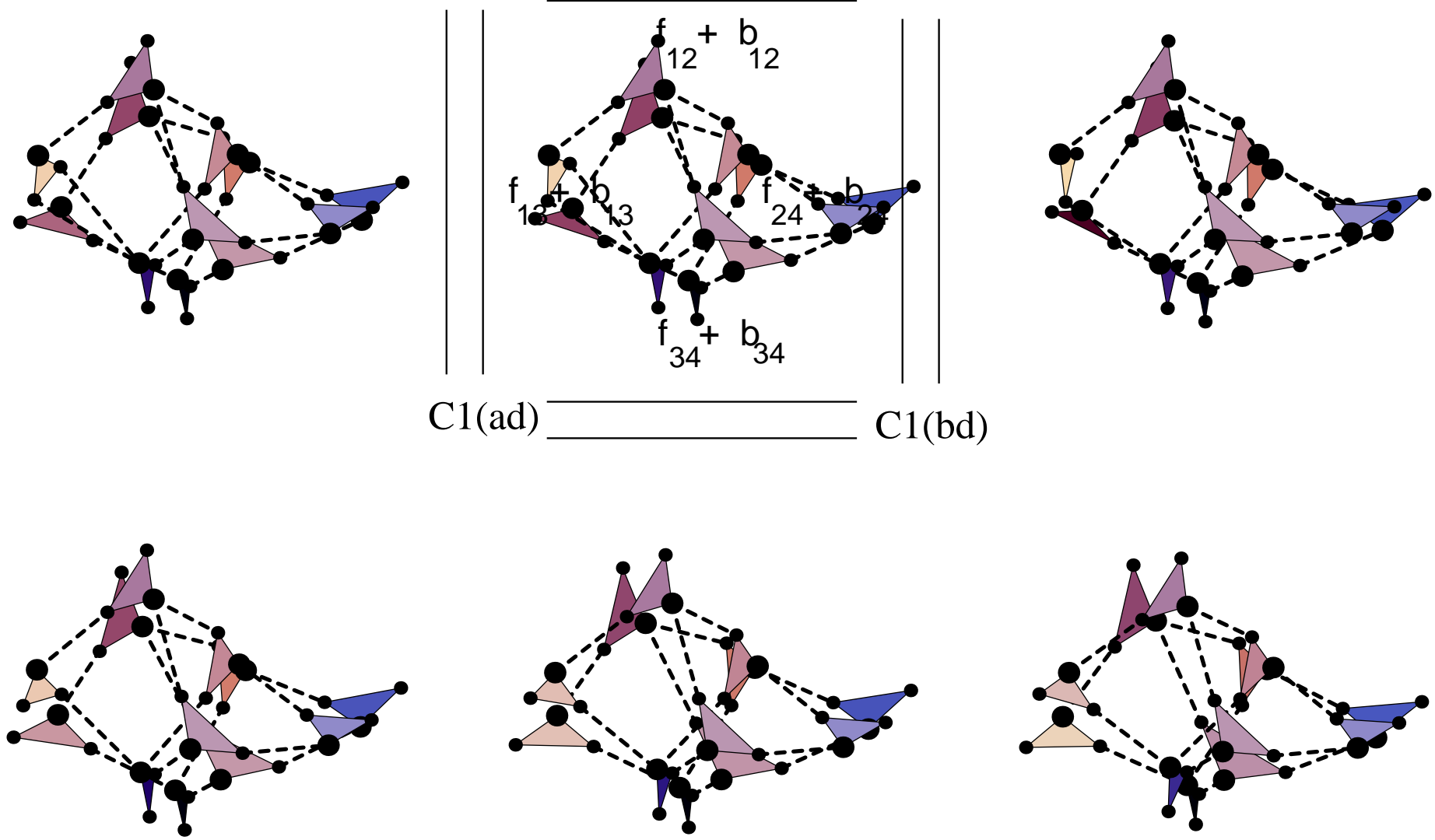